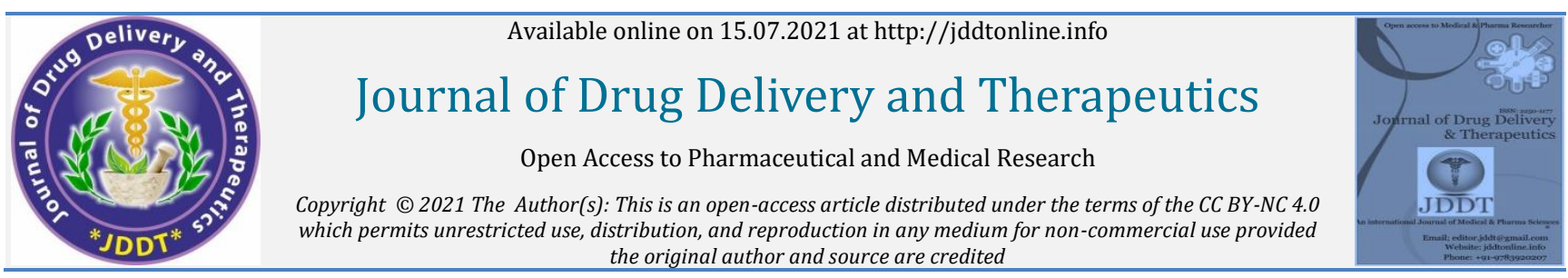

Open Access Full Text Article the original author and source are credited
ting

\title{
A Review on Deprescribing in Elderly
}

\author{
*Christy Babu (D), Lincy George, K. Krishnakumar \\ Department of Pharmacy Practice St James' College of Pharmaceutical Sciences, St James' Hospital Trust Pharmaceutical Research Centre (DSIR \\ recognized), Chalakudy, Kerala, India
}

\begin{tabular}{ll}
\hline Article Info: & \\
\hline & Article History: \\
& Received 12 May 2021 \\
& Accepted 30 June 2021 \\
& Available online 15 July 2021
\end{tabular}

Cite this article as:

Babu C, George L, Krishnakumar K, A Review on Deprescribing in Elderly, Journal of Drug Delivery and Therapeutics. 2021; 11(4):136-137

DOI: http://dx.doi.org/10.22270/jddt.v11i4.4871

*Address for Correspondence:

Christy Babu, Department of Pharmacy Practice St James' College of Pharmaceutical Sciences, St James' Hospital Trust Pharmaceutical Research Centre (DSIR recognized), Chalakudy, Kerala, India.

ORCID ID : https://orcid.org/0000-0002-2224-6349

\begin{abstract}
Deprescribing can be defined as the process of stopping a medication or reducing its dose to reduce adverse effects and improve health outcomes. Elderly people with co morbidities usually have a risk of adverse drug events and these events can only be treated by medicines. Despite the benefits offered by these medicines, older patients are at a high risk of harm caused by these medications. The use of multiple medications for treating the comorbidities may also leads to severe health problems. Deprescribing help to reduce the risk related to inappropriate medicines. Deprescribing is usually a patient centered process which helps to withdraw drugs which are harmful to be prescribed to older patients. Several explicit and implicit tools have been developed internationally to identify these inappropriate medications. Deprescribing of inappropriate medicines would help to reduces drug-related harm and improves the quality of life of older adults.
\end{abstract}

Keywords: Deprescribing, polypharmacy, older adults

\section{INTRODUCTION}

The elderly population can be defined as people aged above 65 and over and by 2050 the proportion of world population over 65 years will nearly double from $12 \%$ to $22 \%$. The rate of population aging is much faster than in the past. The life expectancy of geriatric population seems to be increased because of the advanced health care facility. The physiological and functional capacity of human body would be changed by ageing. This might also alter the pharmacokinetics and pharmacodynamics of drugs leading to clinically relevant consequences to safety and efficacy. The increased prevalence of diseases can influence the pharmacologic effects of drugs. The elderly population has a higher prevalence of chronic and multiple diseases such as cardiovascular diseases, chronic respiratory diseases and so on. ${ }^{1}$

The geriatric population often uses one or more medications for the treatment of co morbidities. The older patients uses an average of two to four medications on a regular basis and poly pharmacy is known to increase the drug-drug or drug-disease interactions. The underuse of medications often reduces benefits and the overuse of medications can cause harm to patients. Inappropriate medication can increase the adverse drug events in geriatrics. Discontinuing or stopping the inappropriate medication would reduce the risk associated with these medicines and which thereby increase the quality of life of geriatric patients.
Deprescribing is an effective method of stopping or discontinuing the medications to reduce the health problems associated with improper medicines. Deprescribing process should be a decision making between physician and patients. The health care professionals should recognize when the medicines need to be stopped and how to deprescribe.It may help to improve the health outcomes in older adults. ${ }^{2}$

\section{NEED FOR DEPRESCRIBING}

\section{Polypharmacy}

Polypharmacy can be defined as the use of multiple medications. These may cause impaired physical and cognitive function. The chances of getting chronic diseases would be increases with age. Several medications may need for the treatment of co morbidities. Polypharmacy occurs when additional medications are prescribed for the treatment adverse effect of another medication. Polypharmacy is known to increase the drug-drug and drug disease interactions. Deprescribing is the best option to reduce these problems as it helps to withdrawing of inappropriate medications.

\section{Fraility}

It is one of the most important problems of geriatrics. The physiological and functional capacity of human body would be changed by ageing. This will further declines homeostatic mechanisms and causes changes in health status, typically delirium. Drugs acting on the central nervous system and 
polypharmacy are the important risk factors for falls. The relationship between falls and cardiovascular drugs is less clear. Antihypertensive drugs also cause orthostatic hypertension and falls in elderly.

\section{Adverse effects}

Elderly people are more vulnerable to adverse drug events because of the changes in the pharmacokinetic and pharmacodynamic properties. Detection of the adverse effects in older patients is a challenge due to changes in the physiological function and cognitive impairment. The adverse effects due to the inappropriate medications can be prevented by stopping or withdrawing the medications.

\section{Lack of effectiveness}

If the risk of the medication overweighs its benefit or if the medication does not shows any effectiveness it is better to discontinue it by the deprescribing process. It may also help to prevent the medicine related harm in elderly. ${ }^{3,4}$

\section{STEPS TO DEPRESCRIBE A MEDICATION}

\section{Prepare the patient for deprescribing}

The first step of the deprescribing process is to get the medication history of the patient. When starting a new drug therapy explains the patient about the benefits of the medication and the drug should be stopped if there any adverse drug reaction occurs. Discuss deprescribing at start of therapy and clarify the doubts of the patient at this time.

\section{Identify any potentially inappropriate polypharmacy}

The second step is to recognize the inappropriate drugs which are taken by the patients. Along with that the adverse drug events, lack of efficacy and changes in treatment goals should also be identified.

\section{Prioritise medicines and wean the dose}

Another important approach is to reduce the dose of the drug. If any adverse drug events suspected, then the inappropriate medication should be discontinued. In the case of polypharmacy, there are different methods to identify the drugs which are apt for deprescribing. Always wean central nervous system-active medicines (especially benzodiazepines, opioids), beta blockers, corticosteroids, levodopa.

\section{Monitor outcomes}

If any withdrawal syndrome occurs, then the medication should be continued and the withdrawal could be done at a slower rate. Monitor the patients regularly to evaluate the positive outcomes of deprescribing and improved cognitive function. ${ }^{5-7}$

\section{DEPRESCRIBING TOOLS}

Deprescribing tools were developed internationally to identify medications which may cause adverse drug events in elderly or that need a review may support the deprescribing process. Most of the deprescribing tools identified were developed by utilizing expert opinion. Assessment of potentially inappropriate medications can be assessed using implicit (judgement-based) or explicit (criteria-based) methods. Each medication is assessed individually and a potentially inappropriate medication (PIM) can be identified.

\section{Implicit tools are:}

- Medication Appropriateness Index(MAI)

- Israel-The Good Palliative Geriatric Practice Algorithm.

\section{Explicit tools are:}

- USA-Beers Criteria

- Canada-Deprescribing guidelines and algorithms

- Ireland-STOPP/START criteria

- Germany-FORTA Criteria

- Germany-PRISCUS Criteria ${ }^{8}$

\section{BENEFITS AND RISK OF DEPRESCRIBING}

Older adults are often willing to withdraw the medication if their doctor recommended to them. It is important to include patients in the deprescribing process. Sometimes patients may be feared about the withdrawal symptoms, but are more willing to have a medicine deprescribe if they know they can restart it if required.

Another barrier of the deprescribing process is the poor communication between the physician and the patients. This again shows the importance of giving information about the risks and benefits of medication use to facilitate an older person's willingness to deprescribe. For example, those who got a brochure which contain the harm from certain drugs and suggested alternative options would significantly more likely to stop the medicine than those who received usual care. ${ }^{9}$

\section{CONCLUSION}

Deprescribing of medications would help to reduce the adverse drug events and may significantly improve the quality of life of older adults. Some medicines can be deprescribed without noticeable effects, while others may shows withdrawal symptoms. These medications need more careful monitoring if they are discontinued. Deprescribing is an effective method to bring down the drug-related harm and would help to enhance the quality of life of older adults. ${ }^{10}$

\section{REFERENCES}

1. Le Bosquet K, Barnett, N Minshull J, Deprescribing: Practical ways to support person-centered, evidence-based deprescribing, Pharmacy, 2019; 7(3):129-139 https://doi.org/10.3390/pharmacy7030129

2. Woodford HJ, Fisher J, New horizons in deprescribing for older people, Age and ageing, 2019; 48(6):768-775.

https://doi.org/10.1093/ageing/afz109

3. Potter K, Flicker L, Page A, Etherton-Beer C, Deprescribing in frail older people: a randomized contolled trail, PloS one, 2016; 11(3):149-155 https://doi.org/10.1371/journal.pone.0149984

4. HilmerSN, GnjidicD, The effects of polypharmacy in older adults, Clinical Pharmacology \& Therapeutics, 2009; 85(1):86-8 https://doi.org/10.1038/clpt.2008.224

5. Le Couteur D, Banks E, Gnjidic D, McLzchlan A, Deprescribing, Australian prescriber, 2011; 34(6):182-185 https://doi.org/10.18773/austprescr.2011.095

6. Liacos M, Page AT, Etherton-Beer C, Deprescribing in older people,Australian prescriber, 2020; 43(4):114-120 https://doi.org/10.18773/austprescr.2020.033

7. Curtin D, Gallagher P,O' Mahony D, Deprescribing in older people approaching end-of-life: development and validation of STOPP Frail version 2,Age and ageing, 2021; 50(2):465-471. https://doi.org/10.1093/ageing/afaa159

8. Planton J, Edlund B, Strategies of reducing polypharmacy in older adults, Journal of gerontological nursing, 2019; 36(1):8-12. https://doi.org/10.3928/00989134-20091204-03

9. Reeve E, To J, Hendrix I, Shakib S, Roberts MS, Wiese MD, Patient barriers to and enablers of deprescribing: a systematic review, Drugs \& ageing, 2013;30(10):793-807. https://doi.org/10.1007/s40266-013-0106-8

10. Salahudeen MS, Deprescribing medications in older people: a narrative review, Drugs of Today, 2018; 54(8):3489-3498. https://doi.org/10.1358/dot.2018.54.8.2856495 